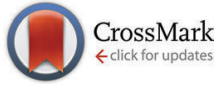

Cite this: Phys. Chem. Chem. Phys., 2015, 17, 18393

Received 2nd April 2015, Accepted 16th June 2015

DOI: $10.1039 / c 5 c p 01937 k$

www.rsc.org/pccp

\section{Imaging phase separation in model lipid membranes through the use of BODIPY based molecular rotors $\dagger$}

\author{
Michael R. Dent, ${ }^{a}$ Ismael López-Duarte, ${ }^{a}$ Callum J. Dickson, ${ }^{a}$ Niall D. Geoghegan, ${ }^{b}$ \\ Jonathan M. Cooper, ${ }^{b}$ Ian R. Gould, ${ }^{a}$ Rob Krams, ${ }^{c}$ James A. Bull, ${ }^{a}$ \\ Nicholas J. Brooks*a and Marina K. Kuimova*a
}

\begin{abstract}
In order to fully understand the dynamics of processes within biological lipid membranes, it is necessary to possess an intimate knowledge of the physical state and ordering of lipids within the membrane. Here we report the use of three molecular rotors based on meso-substituted boron-dipyrrin (BODIPY) in combination with fluorescence lifetime spectroscopy to investigate the viscosity and phase behaviour of model lipid bilayers. In phase-separated giant unilamellar vesicles, we visualise both liquid-ordered $\left(\mathrm{L}_{\mathrm{o}}\right)$ and liquiddisordered $\left(L_{d}\right)$ phases using fluorescence lifetime imaging microscopy (FLIM), determining their associated viscosity values, and investigate the effect of composition on the viscosity of these phases. Additionally, we use molecular dynamics simulations to investigate the orientation of the BODIPY probes within the bilayer, as well as using molecular dynamics simulations and fluorescence correlation spectroscopy (FCS) to compare diffusion coefficients with those predicted from the fluorescence lifetimes of the probes.
\end{abstract}

\section{Introduction}

Diffusion-limited processes are ubiquitous within cell biology, often controlling the rate of mass transport of reagents through a biological system. One of the main parameters controlling the rate of diffusion is the viscosity of the surrounding environment, so precise measurements of intracellular viscosity are necessary in order to fully understand the dynamics of reactions within a cell. ${ }^{1}$ This is particularly true within membrane systems, where viscosity and the subsequent lipid diffusion dynamics play a vital role in the activity of the membrane. ${ }^{2}$ In addition to this, membrane viscosity and heterogeneity are thought to play a role in many important cellular processes, such as cell division, ${ }^{3}$ cell death, ${ }^{4}$ motility ${ }^{5}$ and membrane fusion. ${ }^{6-8}$

There are, however, significant experimental challenges associated with the direct mapping of viscosities and the associated diffusion coefficients to a high degree of spatial and temporal resolution within a biological membrane. Fluorescence based techniques provide a non-invasive way of investigating membranes,

\footnotetext{
${ }^{a}$ Department of Chemistry, Imperial College London, Exhibition Road, London, SW7 2AZ, UK. E-mail: m.kuimova@imperial.ac.uk, nicholas.brooks@imperial.ac.uk

${ }^{b}$ Division of Biomedical Engineering, School of Engineering, University of Glasgow, Oakfield Avenue, Glasgow, G12 8LT, UK

${ }^{c}$ Department of Bioengineering, Royal School of Mines Building, Imperial College London, Exhibition Road, London, SW7 2AZ, UK

$\dagger$ Electronic supplementary information (ESI) available. See DOI: 10.1039/c5cp01937k
}

and in recent years methods such as fluorescence recovery after photobleaching (FRAP) ${ }^{9,10}$ and fluorescence correlation spectroscopy (FCS) ${ }^{11}$ have been used successfully to probe diffusion within a biological membrane. Whilst these techniques can accurately determine diffusion coefficients within a membrane, they often cannot provide detailed spatial and temporal information.

An emerging technique for measuring microscale viscosity within a membrane system is through the use of molecular rotors, synthetic organic fluorophores whose fluorescence properties are dependent on the friction imparted on them by the surrounding environment. ${ }^{1,12}$ Upon excitation with a photon, a molecular rotor can either undergo radiative decay via fluorescence emission or decay via a nonradiative pathway, typically involving an intramolecular rotation mechanism. The rate of this nonradiative decay is influenced by the friction imposed by the surrounding environment, i.e. the local microviscosity. This competition between radiative and nonradiative decay leads to fluorescence quantum yield and lifetime increasing sharply with increasing viscosity. ${ }^{1}$ This can be described using the Förster-Hoffmann equation: ${ }^{13}$

$$
\Phi_{\mathrm{f}}=z \eta^{\alpha}
$$

where $\Phi_{\mathrm{f}}$ is the fluorescence quantum yield, $\eta$ is the viscosity of the surrounding environment, and $z$ and $\alpha$ are constants.

Whilst this approach is useful in bulk systems where concentration is known, in heterogeneous systems like biological membranes it becomes impossible to determine whether changes in 
fluorescence intensity are due to a change in viscosity or arise because of a variation in local fluorophore concentration. This issue could be addressed using a ratiometric approach, ${ }^{14-17}$ or through the use of fluorescence lifetime, ${ }^{18-21}$ with both methods providing a concentration bias-free method of determining the surrounding viscosity.

The lifetime of a molecular rotor can be related to the viscosity of the surrounding environment using a variant of the FörsterHoffmann equation: ${ }^{13,21}$

$$
\log \tau_{\mathrm{f}}=\log \left(\frac{z}{k_{\mathrm{r}}}\right)+a \log \eta
$$

where $\tau_{\mathrm{f}}$ is the fluorescence lifetime and $k_{\mathrm{r}}$ is the radiative decay constant.

Fluorescence lifetime imaging microscopy (FLIM) can be used to generate an image of fluorescence lifetimes across a heterogeneous sample. ${ }^{22}$ By using FLIM in conjunction with molecular rotors it is therefore possible to produce an image displaying the different viscosities across a heterogeneous sample.

Molecular rotors based on meso-substituted boron dipyrrin (BODIPY) dyes are known to exhibit a strong dependence of their fluorescence lifetime on the viscosity of the surrounding medium. $^{20,21,23,24}$ The fluorescent BODIPY core carries the advantage of being chemically robust, allowing a wide range of potential modifications to the meso-phenyl ring without degradation of the dye. ${ }^{25}$ Increasing viscosity leads to a partial restriction of the rotation of the meso-phenyl ring, which causes a decrease in the rate of non-radiative decay, increasing both the fluorescence lifetime and quantum yield. ${ }^{26}$

BODIPY based molecular rotors have previously been used to quantitatively assess viscosity across a range of systems, including microbubbles, ${ }^{27}$ bacterial spores ${ }^{28}$ and live cells. ${ }^{1,29}$

BODIPY rotors $\mathbf{1}^{21}$ and $2^{20}$ (Scheme 1 ) have been synthesised previously, and both their quantum yield and fluorescence lifetime have been shown to increase according to the Förster-Hoffmann equation within a range of $c a .10-1000 \mathrm{cP}$ in calibration mixtures of methanol and glycerol.

BODIPY rotor 1 has previously ${ }^{19}$ been shown to be membrane soluble, and has been used to determine the viscosities associated with different phases within artificial bilayer systems. A sharp change in viscosity at the gel transition temperature of a saturated lipid was observed, as well as the different viscosities within coexisting liquid domains in ternary bilayers. Liquid-ordered $\left(\mathrm{L}_{\mathrm{o}}\right)$ phases were found to display a higher viscosity than liquiddisordered $\left(\mathrm{L}_{\mathrm{d}}\right)$ phases, owing to the increased molecular
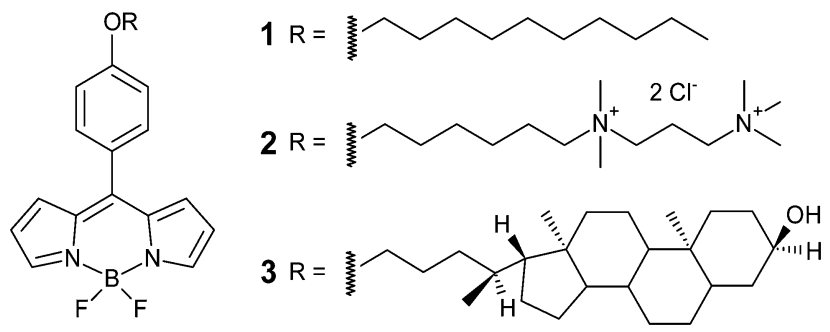

Scheme 1 Structure of the three BODIPY rotors. packing and rigidity within the $\mathrm{L}_{\mathrm{o}}$ phase. The probe was, however, found to partition very weakly into the $\mathrm{L}_{\mathrm{o}}$ phase, affecting the quality of the data collected from this phase. In addition to this, when introduced into human cancer cells in an attempt to visualise plasma membrane viscosity, BODIPY 1 was found to rapidly internalise, hence it could only be used to determine the viscosity of the lipid membranes of internal cellular organelles. ${ }^{21}$

BODIPY rotor 2 , however, has recently been shown to target the plasma membrane of the cell, ${ }^{20}$ due to the double positive charge reducing the rate of endocytosis, and has shown an average plasma membrane viscosity of $c a .270 \mathrm{cP}$ for SK-OV-3 cancer cells. It has not yet been used to give information on the lipid order and heterogeneity within model membrane systems.

Here we investigate BODIPY rotors 1-3 (Scheme 1), where rotor 3 was designed with a cholestanol moiety with the aim of targeting cholesterol rich regions within plasma membranes of live cells. A variety of cholesterol derivatives of BODIPY dyes have been previously synthesised ${ }^{30-32}$ and some have even been reported ${ }^{33}$ to target $\mathrm{L}_{\mathrm{o}}$ phases in artificial bilayers. Cholestanol has been found to interact in a similar way to cholesterol inside lipid bilayers, albeit with weaker interactions. ${ }^{34,35}$

In this study we report the synthesis and full characterisation of BODIPY 3. We compare the use of the three BODIPY molecular rotors (1-3) as probes of lipid bilayers, using their fluorescence lifetime to investigate the viscosity and ordering within the bilayers. In addition to this, we use molecular dynamics simulations and FCS to investigate the diffusional properties of the three rotors within lipid bilayers in order to relate this to viscosity values calculated from the fluorescence lifetime of the probe.

\section{Materials and methods}

All solvents were purchased from Sigma-Aldrich or VWR and used without further purification. All lipids were obtained from Avanti Polar Lipids.

The meso-substituted dyes BODIPY 1 and 2 were synthesised as described elsewhere. ${ }^{19,20}$ The synthesis of BODIPY rotor 3 is outlined in Scheme 2. Firstly, commercially available lithocholic acid was reacted with an excess of tert-butyldiphenylsilyl chloride to give bis-silyl protected derivative $\mathbf{8}$ in $70 \%$ yield. Reduction of the silyl ester to the alcohol derivative was achieved with $\mathrm{LiAlH}_{4}$ to afford compound 7 quantitatively, which was further transformed into the corresponding sulfonate ester 6 by the use of methanosulfonyl chloride in the presence of triethylamine. The next step required the reaction of mesylate 6 with 4-hydroxybenzaldehyde to give aldehyde $\mathbf{5}$ in good yield. The removal of the silyl protecting group to afford derivative 4 was achieved by the use of TBAF in THF at $40{ }^{\circ} \mathrm{C}$. Finally BODIPY rotor 3 was obtained via condensation of compound 4 with an excess of pyrrole to give the corresponding dipyrromethane, which was further reacted with DDQ and followed by treatment with $\mathrm{BF}_{3} \cdot \mathrm{OEt}_{2}$. After purification by column chromatography, BODIPY rotor 3 was isolated in $28 \%$ yield from aldehyde 4 . $^{36}$

Absorption spectra were obtained using an Agilent 8452 UV/Vis spectrometer, and emission spectra using a Horiba Jobin 


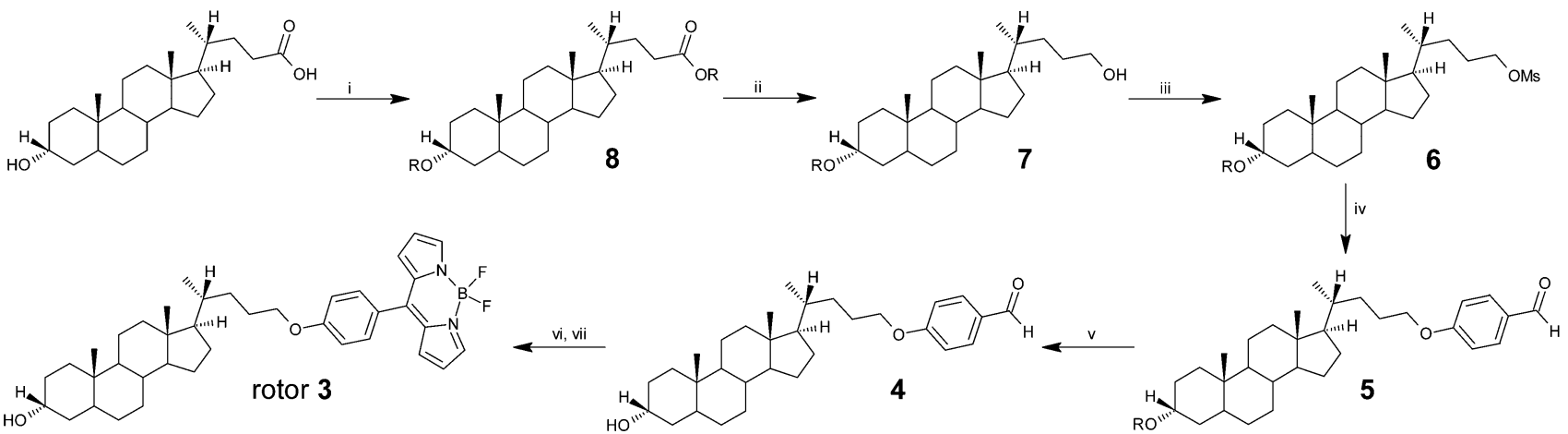

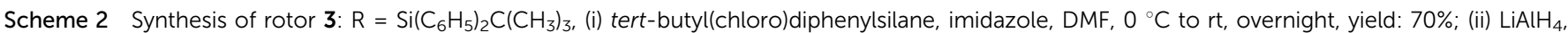
THF, $0{ }^{\circ} \mathrm{C}$ to rt, overnight, yield: $92 \%$; (iii) $\mathrm{MsCl}, \mathrm{Et}_{3} \mathrm{~N}, \mathrm{CH}_{2} \mathrm{Cl}_{2}, \mathrm{O}{ }^{\circ} \mathrm{C}$ to rt, $5 \mathrm{~h}$, yield: $90 \%$; (iv) 4-hydroxybenzaldehyde, $\mathrm{K}_{2} \mathrm{CO}_{3}, \mathrm{DMF}^{\circ} 80{ }^{\circ} \mathrm{C}, 5 \mathrm{~h}, \mathrm{yield}$ : $86 \%$; (v) TBAF, THF, $40{ }^{\circ} \mathrm{C}, 12 \mathrm{~h}$, yield: $69 \%$; (vi) neat pyrrole, TFA, rt, $1 \mathrm{~h}$, yield: $94 \%$; (vii) DDQ, $\mathrm{CH}_{2} \mathrm{Cl}_{2}, \mathrm{rt}, 1 \mathrm{~h}$ and then $\mathrm{BF}_{3} . \mathrm{OEt}_{2}, \mathrm{Et}_{3} \mathrm{~N}, \mathrm{CH}_{2} \mathrm{Cl}_{2}, \mathrm{rt}$, overnight, yield: $28 \%$. Detailed synthetic information is given in Section (6) of the ESI. $\dagger$

Yvon Fluoromax 4. Lifetime measurements were obtained using a Horiba Jobin Yvon IBH $5000 \mathrm{~F}$ time-correlated single photon counting (TCSPC) device, using a pulsed NanoLED source at either 404 or $467 \mathrm{~nm}$. All TCSPC measurements gave 10000 counts in the peak channel, and DAS software was used for fitting the decays. Decays were established as biexponential if a monoexponential fit to the data gave a $\chi^{2}$ value of 2 or above and fitting with a biexponential function improved the quality of the fit (as indicated by a lower $\chi^{2}$ value). Unless otherwise stated, all spectra were taken at $298 \mathrm{~K}$.

All bilayer studies used a maximum BODIPY rotor concentration of $0.5 \mathrm{~mol} \%$ ( $1: 200$ rotor: lipid) to prevent dye aggregation ${ }^{19,27}$ and to avoid significant disruption of the bilayer structure. Large unilamellar vesicles (LUVs) were formed using the gas extrusion method. A solution of the appropriate lipid and BODIPY rotor was prepared in chloroform, which was then evaporated off under nitrogen. Multilamellar vesicles (MLVs) were then prepared by hydrating the lipid film using enough water to give a $1 \mathrm{mmol} \mathrm{dm}{ }^{-3}$ solution of lipid and vortexing for 1 minute above the gel transition temperature of the lipid. This was then extruded 10 times through a polycarbonate membrane with a pore diameter of $200 \mathrm{~nm}$ using a LIPEX extruder (Northern Lipids Inc., Canada), ensuring the solution was kept above the gel transition temperature of the lipid.

Giant unilamellar vesicles (GUVs) were prepared using the electroformation method. ${ }^{37} \mathrm{~A}$ solution of $1 \mathrm{mg} \mathrm{ml}^{-1}$ lipid with the appropriate BODIPY rotor in chloroform was added dropwise (ca. $30 \mu \mathrm{L}$ ) onto a clean conductive indium tin oxide (ITO) coated glass slide and spread using a glass coverslip to give a thin film of lipid and BODIPY rotor, before being lyophilised for 60 minutes. A polydimethyl siloxane (PDMS) spacer was placed over the lipid film and a second ITO coated slide was then placed on top of the spacer, creating a sealed chamber. The chamber was filled with $0.1 \mathrm{~mol} \mathrm{dm}^{-3}$ aqueous sucrose solution using a syringe. An AC voltage of $1.0 \mathrm{~V}$ and $10 \mathrm{~Hz}$ was applied for 90 minutes, making sure the chamber was held above the gel transition temperature of the lipids being used. In order to be visible in phase contrast microscopy, the GUVs formed in this way were suspended in a $0.125 \mathrm{~mol} \mathrm{dm}^{-3}$ aqueous solution of glucose.
FLIM measurements were performed on a Leica TSC SP5 II inverted confocal microscope with a $63 \times$ water immersion objective (NA = 1.2). A Coherent Chameleon Vision II modelocked femtosecond Ti : sapphire laser was used for excitation, using two-photon excitation at $900 \mathrm{~nm}$. Decay traces were analysed using SPCimage software, which was used to fit decay traces in order to give lifetime values. All images were binned to give a minimum of 100 counts per pixel before fitting.

Molecular dynamics simulations were performed using the AMBER $^{38}$ suite using lipid parameters discussed elsewhere ${ }^{39}$ (see $\mathrm{ESI} \dagger$ (1) for a detailed description of the simulations). Briefly, diffusion of the three dyes inside a 1,2-dioleoyl-sn-glycero-3phosphocholine (DOPC) bilayer was simulated at a range of temperatures and the preferred position of BODIPY rotor 1 in a 1,2-dipalmitoyl-sn-glycero-3-phosphocholine (DPPC) bilayer was simulated at $293 \mathrm{~K}$, meaning the bilayer was in a gel phase. To obtain the preferred orientation of the rotors within the bilayer, each dye was placed in the water phase above the bilayer and the system was simulated for $100 \mathrm{~ns}$ at $303 \mathrm{~K}$, during which time each dye diffused into the bilayer. This system was then minimised and heated to the desired temperature in order to simulate the diffusion coefficients of the rotors within the bilayer.

Fluorescence correlation spectroscopy (FCS) was performed using the Becker and Hickl DCS-120 confocal laser scanning system. The excitation light of a $473 \mathrm{~nm}$ pulsed diode laser, repetition rate $50 \mathrm{MHz}$, was focussed to the sample via a $40 \times$ 1.2 NA Zeiss C-Apochromat water immersion objective. The resultant fluorescence was collected by the same objective and focussed via a $250 \mu \mathrm{m}$ pinhole to a HPM-100-40 Hybrid Photo Multiplier Tube (PMT) module, free of afterpulsing effects. The system was optimised to account for possible aberrations as outlined in ref. 40 . The lateral radius, $\omega_{0}$, of the confocal volume was calibrated using a $10 \mathrm{nM}$ concentration of fluorescein in water and found to be $262 \mathrm{~nm}$. To determine the diffusion coefficients of the three molecular rotors in DOPC GUVs, the central point of the focal volume was positioned to the top side of the vesicles determined by the maximal detected fluorescence signal. Short correlation measurements of 10 seconds were recorded to minimise any potential artefacts arising from photobleaching 
or vesicle undulation. Four vesicles were measured 10 times for each rotor at temperatures of $293 \mathrm{~K}, 313 \mathrm{~K}$ and $333 \mathrm{~K}$. The measured auto-correlation function $G(\tau)$ was fit with a model describing 2D diffusion to determine the diffusion coefficient, $D$, as outlined elsewhere ${ }^{40}$ shown by eqn (3).

$$
G(\tau)=\frac{1}{\langle N\rangle} \frac{1}{\left(1+\frac{\tau}{\tau_{\mathrm{D}}}\right)}
$$

Where $\langle N\rangle$ is the average number of diffusing molecules in the focal volume, $\tau$ is the correlation time and $\tau_{\mathrm{D}}$ is the diffusion time related to the diffusion coefficient, $D$, by the relationship $\tau_{\mathrm{D}}=\frac{\omega_{0}^{2}}{4 D}$.

\section{Results and discussion}

\section{Photophysical characterisation of the BODIPY rotors}

The Förster-Hoffmann calibration of lifetime against viscosity for BODIPY rotors 1 and 2 have been reported previously, ${ }^{19,20}$ (Fig. S3, ESI $\dagger$ ) and the data for BODIPY rotor 3 is shown in Fig. 1. The calibration measurements were performed in methanolglycerol mixtures at a range of temperature, giving a viscosity range of between 10-1000 cP.

The graph shown in Fig. 1b shows a linear dependence of lifetime against viscosity on a double logarithmic scale within the viscosity range tested, verifying that BODIPY rotor 3 behaves as a molecular rotor, and shows good agreement with the FörsterHoffmann equation within this viscosity range. In addition to this, the fact that the lifetime values do not deviate significantly from the linear correlation, even for results obtained at different temperatures, suggests that the photophysical properties of the rotor are unaffected by temperature, consistent with previous results for rotors $\mathbf{1}^{19}$ and $2 .^{20}$

The emission and absorption spectra of the three rotors (Fig. S4, $\left.\mathrm{ESI}^{\dagger}\right)$ are almost identical, suggesting that the electronic structures of the dyes are very similar, and that the different groups attached to the rotors do not affect their fluorescence properties.

Several previously studied molecular rotors ${ }^{41}$ have been shown to display highly polarity sensitive emission. This could potentially limit their usefulness as probes of heterogeneous environments where the polarity of the surrounding environment is unknown.

At low viscosities, the lifetime of BODIPY rotors is influenced by solvent polarity (Fig. S5, ESI $\dagger$ ). We hypothesised, similarly to Haidekker et $a .^{41}$ that any effect of solvent polarity would be negligible at high viscosities, and in order to test this, we compared the calibration plot of BODIPY rotor 1 obtained in methanol/ glycerol, two highly polar solvents, with fluorescence lifetimes of rotor $\mathbf{1}$ in castor oil, a viscous and very nonpolar solvent, at different temperatures. The results are shown in Fig. 2. The lifetimes of rotor $\mathbf{1}$ in castor oil show excellent overlap with the methanol/glycerol calibration plot, which suggests that solvent polarity has little influence on the fluorescence lifetime of BODIPY rotors at high viscosities. Fig. S6 (ESI $\dagger$ ) shows the fluorescence lifetime of rotor 1 in 1,2-pentanediol, 1,2-butanediol and 1,3-propanediol at viscosities between 10-100 $\mathrm{cP}$, in order to
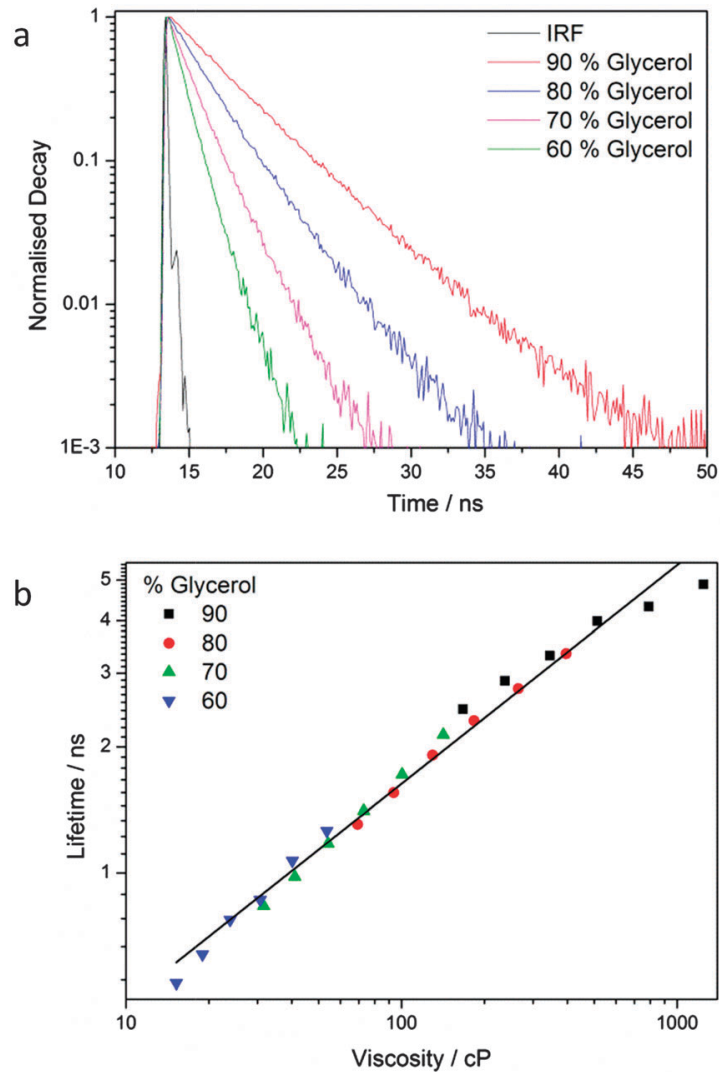

Fig. 1 (a) Fluorescence decays of rotor $\mathbf{3}$ in methanol-glycerol mixtures of different viscosity, (b) Förster-Hofmann plot for rotor 3 in methanolglycerol mixtures measured at $5-55^{\circ} \mathrm{C}$ on a log-log scale (see Fig. S3, ESI $\dagger$ for data for the rotors 1 and 2).

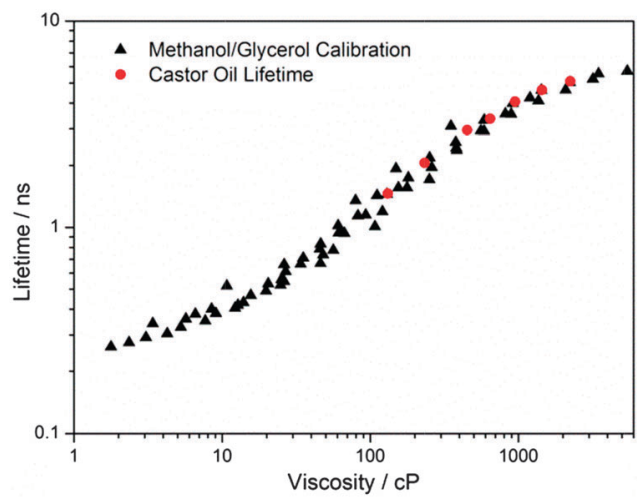

Fig. 2 Calibration plot for rotor 1 (adapted from ref. 19) overlaid with fluorescence lifetimes of rotor 1 recorded between $10-60{ }^{\circ} \mathrm{C}$ in castor oil.

demonstrate the effect of polarities at low viscosities. Up to ca. $80 \mathrm{cP}$, there appears to be an effect of solution polarity on BODIPY lifetime, but beyond that, the lifetime values overlap well, suggesting that polarity is playing less of a role in determining the fluorescence lifetime.

It should be noted that castor oil is intrinsically fluorescent, resulting in biexponential decays for solutions of rotor $\mathbf{1}$ in castor oil (Fig. S7 and S8, ESI $\dagger$ ). The autofluorescence from castor oil contributed a small percentage of the total signal 
(ca. $20 \%$ at room temperature), with a lifetime that did not change with temperature.

\section{Viscosity studies in large unilamellar vesicles}

In order to test the three BODIPY rotors as probes of membrane viscosity, they were incorporated into large unilamellar vesicles (LUVs) with $200 \mathrm{~nm}$ diameter made up of DOPC, an unsaturated lipid, DPPC or egg yolk sphingomyelin (EYSM), saturated lipids. At room temperature rotors $\mathbf{1}$ and $\mathbf{3}$ were found to give monoexponential decay traces in DOPC, and biexponential decays in the saturated lipids (see Fig. S9, ESI $\dagger$ for fluorescence decays of rotors 1 in DOPC, DPPC and EYSM, and rotor 2 in DOPC and EYSM). Rotor 2 in DOPC gave a biexponential decay, with one component showing a very short lifetime on the order of $500 \mathrm{ps,}$ and one component similar to the lifetimes of rotors $\mathbf{1}$ and $\mathbf{3}$ in DOPC. Rotor 2 is water soluble, and since the short lifetime component matched the lifetime of rotor 2 in the aqueous phase, it was attributed to the small percentage of the rotor partitioned in the water phase (15\% of the decay, see Fig. S9, ESI $\dagger$ ). The long lifetime component was treated as the viscosity-dependent lifetime of the probe in the DOPC bilayer.

The calculated viscosities obtained in DOPC bilayers with rotors 1-3 at a range of temperatures are shown in Fig. 3. This result strongly suggests that the viscosity sensitive part of the probe resides in the same part of the bilayer for each rotor, and that BODIPY rotors are reliable probes for determining membrane viscosity in disordered liquid membranes.

As temperature increases, the measured lifetime decreases, indicating a reduction in the bilayer viscosity. The calculated viscosities accessible with the three BODIPY rotors shown in Fig. 3 overlap very well over the biologically relevant viscosity range of $c a$. 20-500 $\mathrm{cP} .^{18,20,21,28}$ However, it should be noted that a high degree of lipid ordering has been previously reported within cell membranes using polarity-sensitive probes. ${ }^{42,43}$

Fluorescence decay traces of rotor 3 in DOPC, DPPC and EYSM LUVs at room temperature are shown in Fig. 4a. The decay in DOPC is clearly monoexponential, whereas the decays in gel phase DPPC and EYSM are biexponential. The effect of changing temperature on the fluorescence lifetime derived viscosity of rotor 3 in DPPC LUVs is shown in Fig. 4 b. Above $41^{\circ} \mathrm{C}$, the gel

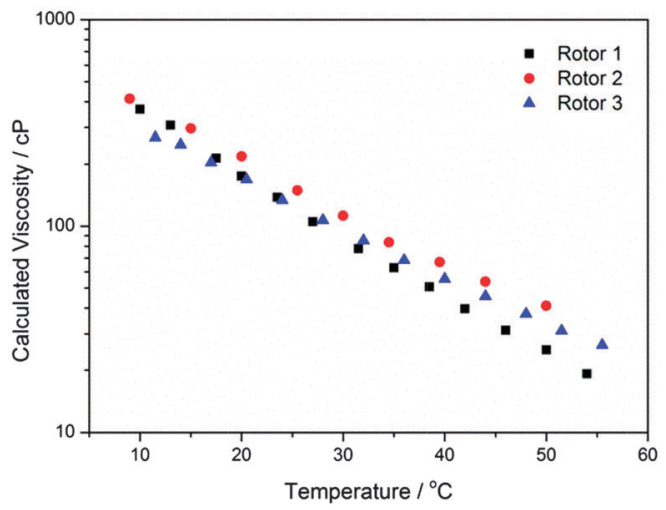

Fig. 3 Viscosities calculated from fluorescence lifetimes measured for the three BODIPY rotors in DOPC LUVs between $9-55^{\circ} \mathrm{C}$. transition temperature of DPPC, the LUVs display similar viscous properties to DOPC, exhibiting monoexponential fluorescence decays. However, below this temperature, the decay traces become biexponential. The two lifetimes extracted from these decays are shown in Fig. 4b, plotted against temperature. For $\tau_{2}$ against temperature, there is a very sharp change in the gradient below $41{ }^{\circ} \mathrm{C}$, suggesting that there is a change in phase. A similar pattern is observed for EYSM. The biexponential decays of the BODIPY probes in gel phase bilayers have previously ${ }^{19,44}$ been attributed to the rotors taking on two conformations within the bilayer, showing two distinct 'viscosities', as the two environments hinder the intramolecular rotation of the non-radiative decay process differently. It was asserted that the presence of two components is concentration independent and is as such not due to aggregation of the rotor. ${ }^{19}$

Finally, the effect of cholesterol on the viscosity of lipid bilayers was investigated. Fig. 4c and d show the effect of adding cholesterol on the fluorescence lifetime of rotor 3 within DOPC and EYSM LUVs upon varying temperature. The addition of cholesterol to the bilayer caused a significant decrease in the viscosity of EYSM LUVs, but an increase in the viscosity of DOPC LUVs. Increasing the amount of cholesterol from $10 \%$ up to $30 \%$ had little effect on the viscosity of DOPC LUVs, but further decreased the viscosity within EYSM LUVs. It is well established that cholesterol increases the ordering of unsaturated lipids, and decreases ordering of saturated lipids, ${ }^{45}$ and one of the main roles of cholesterol within the plasma membrane is to mediate lipid ordering, ${ }^{46}$ so it is perhaps not surprising that the addition of cholesterol brings the viscosities of EYSM and DOPC closer to each other. BODIPY rotors in the gel phase have been shown to exhibit biexponential fluorescence decays (Fig. 4), due to multiple conformations or environments for the dye and it may be a similar effect that results in biexponential decays observed for BODIPY in DOPC/Chol LUVs.

For saturated lipids such as EYSM, the addition of cholesterol disrupts the tight packing of the gel phase lipid molecules, forming a liquid-ordered phase, ${ }^{47}$ which can be seen in Fig. 4 , where the addition of cholesterol prevents the sharp change in viscosity associated with the gel transition temperature of EYSM. Cholesterol is also known to condense unsaturated lipids such as DOPC, ${ }^{48}$ increasing the viscosity and lipid order, which is consistent with the results of our experiments.

\section{The position of BODIPY rotors within the bilayer}

In order to be able to reliably use a molecular rotor as a probe of membrane viscosity it is essential to know its position in the bilayer. Due to various intramolecular interactions, the lateral pressure varies greatly between the head and tail groups of the lipids within the bilayer, ${ }^{49}$ meaning that resistance to intramolecular rotation of a molecular rotor will be different in the tail region of the lipid bilayer compared with the head groups. Therefore, different molecular rotors that take on different orientations may give different viscosity values for the same bilayer.

In order to determine the localisation of the BODIPY probes, the molecular dynamics program $\mathrm{AMBER}^{38}$ was used to simulate the behaviour of the BODIPY rotors within DOPC 


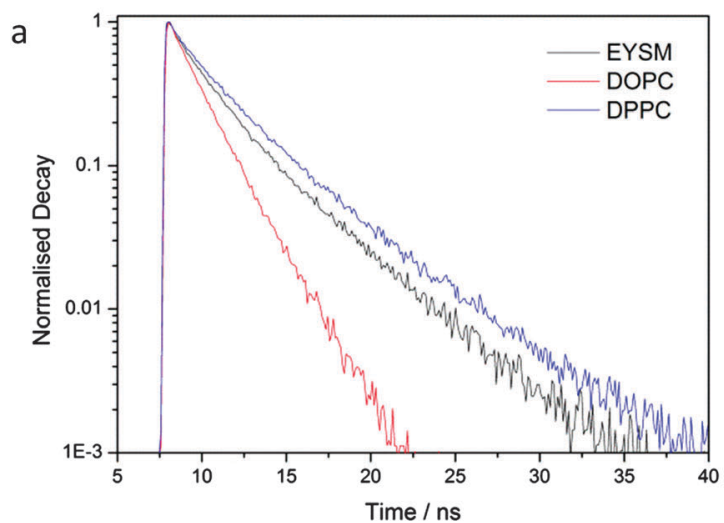

b

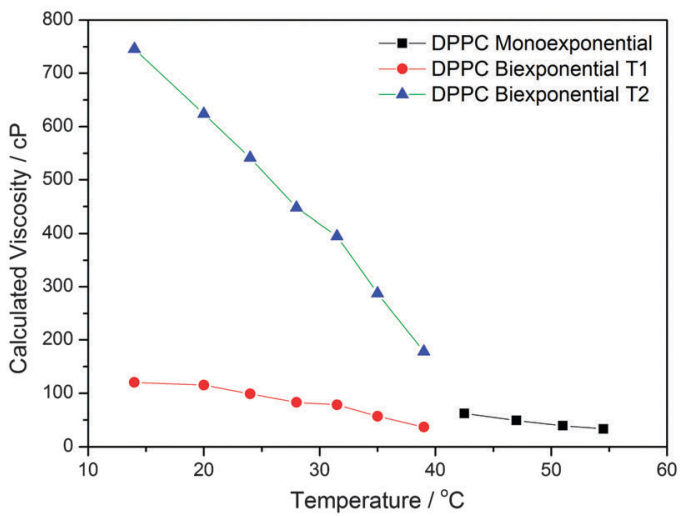

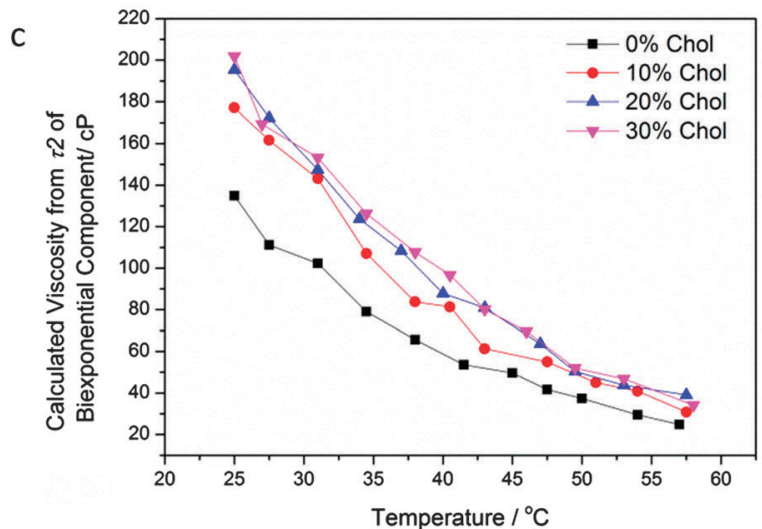

d

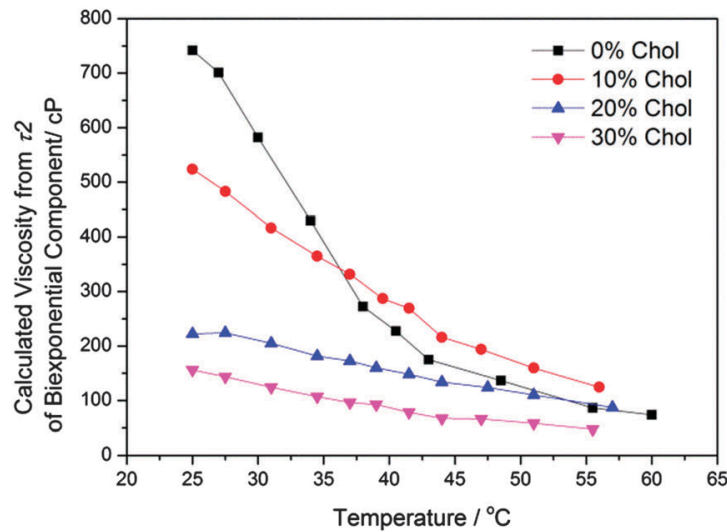

Fig. 4 (a) Fluorescence decays of rotor 3 in DOPC, DPPC and EYSM, (b) calculated viscosity against temperature of rotor 3 in DPPC, highlighting the gel transition temperature (see Fig. S10, ESI $\dagger$ for related data for rotors 1 and $\mathbf{2}$ in DPPC), (c) calculated viscosity of rotor $\mathbf{3}$ in DOPC with varying levels of cholesterol at a range of temperatures, (d) calculated viscosity of rotor $\mathbf{3}$ in egg yolk sphingomyelin (EYSM) with varying levels of cholesterol at a range of temperatures.

and DPPC bilayers, with the final orientations of the dyes shown in Fig. 5.

The simulations showed that the head groups of the BODIPY rotors are similar distances from the core of the bilayer for each of the three rotors in DOPC, see Fig. S2 (ESI $\dagger$ ) for the calculated electron density profiles for each rotor. Due to this similarity in electron density profiles and also due to very similar viscosities reported by 1-3 in DOPC bilayers (Fig. 3) and DPPC bilayers, including upon a liquid to gel phase transition (Fig. 4 and Fig. S10, ESI $\dagger$ ), we can deduce that it is primarily the head group of the BODIPY rotors that is responsible for their viscosity sensitivity. The fact that the dyes take on one preferred orientation is consistent with the monoexponential fluorescence decays observed for the rotors in DOPC bilayers.

For the DPPC simulation, two preferred orientations for BODIPY rotor 1 were found, which again correlates with the fact that BODIPY rotors exhibit biexponential decays within gel-phase lipid systems. This agrees with the fluorescence anisotropy study performed by Olšinova et $a l .{ }^{44}$ on BODIPY rotors in gel phase bilayers, which suggested that the rotors adopt two orientations: one perpendicular and one parallel to the membrane normal.
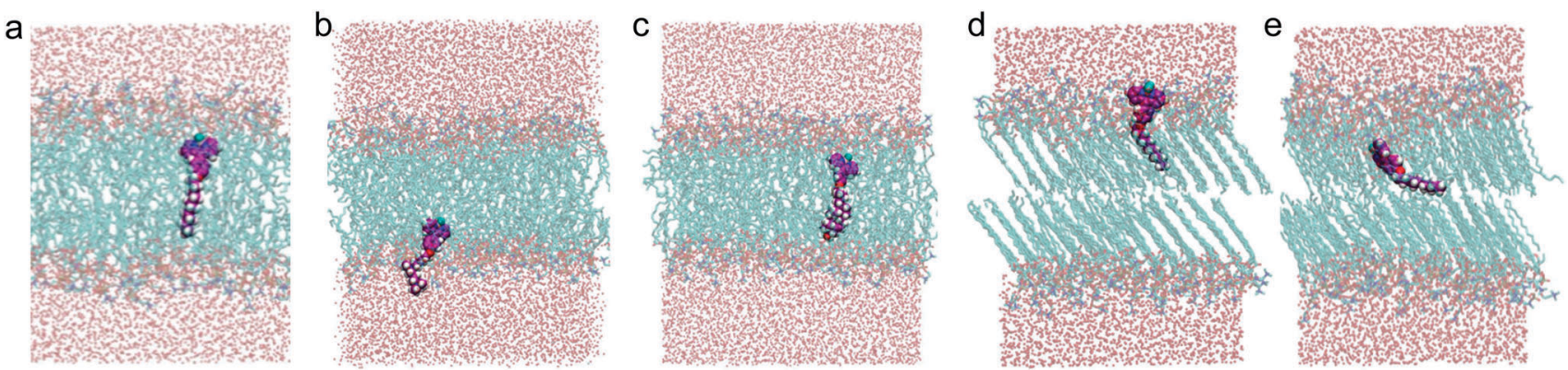

Fig. 5 Molecular dynamics simulations showing the orientations of (a) rotor $\mathbf{1}$, (b) rotor $\mathbf{2}$, and (c) rotor $\mathbf{3}$ in a DOPC bilayer, and (d) and (e) the two orientations of rotor 1 in a gel phase DPPC bilayer. Simulated DOPC bilayers have a membrane thickness of $37.0 \pm 0.2 \AA$ and DPPC bilayers have a membrane thickness of $37.9 \pm 0.5 \AA^{39}$ 
Overall, the molecular dynamics simulations suggest that rotors 1-3 behave in a similar manner within lipid bilayers, independent of the nature of the $\mathrm{R}$ group. In addition to this, the simulations show that the BODIPY rotors intercalate within the lipid molecules within the bilayer, rather than reside in a cavity between the two leaflets of the bilayer.

\section{Estimating diffusion coefficients within bilayers}

A potential drawback in the use of methanol:glycerol solutions of known viscosity for the calibration of BODIPY rotors is that a homogeneous bulk solution with free diffusion in three dimensions may not be a good representation of a lipid bilayer, a quasi-twodimensional systems with a high degree of heterogeneity.

It should also be noted that 'viscosity' is a macroscopic property, and is somewhat poorly defined for a heterogeneous microscale system such as a lipid bilayer. The fluorescence lifetime of a molecular rotor in a lipid bilayer will be affected by the lipid packing of the surrounding environment, giving an idea of the 'microviscosity' of the surrounding environment. ${ }^{44}$ Higher membrane viscosities will correspond to lower diffusion coefficients of species within the membrane.

Using FCS, it is possible to measure two-dimensional diffusion coefficients for fluorescent probes within membranes, ${ }^{50}$ which can then be related to viscosity values through the wellestablished Saffman-Delbrück formula: ${ }^{51}$

$$
D_{\mathrm{sd}}=\frac{k_{\mathrm{B}} T}{4 \pi \eta_{\mathrm{m}} h}\left[\ln \left(\frac{2 L_{\mathrm{sd}}}{a}\right)-\gamma\right]
$$

where $D_{\text {sd }}$ is the Saffman-Delbrück diffusion coefficient, $a$ is the radius of the membrane inclusion, $h$ is the bilayer thickness, $\eta_{\mathrm{m}}$ is the membrane viscosity, $\gamma$ is the Euler-Mascheroni constant $(\gamma \approx 0.577)$, and $L_{\text {sd }}$ is the Saffman-Delbrück length, given by:

$$
L_{\mathrm{sd}}=\frac{h \eta_{\mathrm{m}}}{2 \eta_{\mathrm{f}}}
$$

where $\eta_{\mathrm{f}}$ is the viscosity of the surrounding fluid. It should, however, be noted that this formula makes a number of assumptions, including assuming that the membrane inclusion is cylindrical and that the membrane is homogeneous, and so may not accurately reflect the environment experienced by a molecular fluorophore.

We have used three complementary methods to estimate diffusion coefficients in bilayers. Firstly, we calculated the diffusion coefficient from the viscosity values obtained using the lifetimes of the BODIPY rotors 1-3 and the Saffman-Delbrück equation for DOPC bilayers. Secondly, diffusion coefficients were obtained from performing FCS on rotors 1-3 in DOPC GUVs, and finally molecular dynamics simulations of the three rotors in DOPC bilayers were used to calculate diffusion coefficients. The diffusion coefficients calculated using the three methods for rotors 1-3 at a range of temperatures in DOPC bilayers are shown in Table 1 . See Table S2 (ESI $\dagger$ ) for the parameters used for the Saffman-Delbrück calculations.

The diffusion coefficient values are very similar for each of the methods used; overlapping particularly well at $313 \mathrm{~K}$. There is particularly good agreement between FCS and the molecular dynamics simulations, typically within $1 \mu \mathrm{m}^{2} \mathrm{~s}^{-1}$ of each other suggesting that the simulations are an accurate reflection of real membrane systems under these conditions. Whilst the diffusion coefficients calculated using the Saffman-Delbrück equation are less accurate, they are still generally within a factor of two of the FCS and molecular dynamics simulations, which, given the inherent inaccuracies of the Saffman-Delbrück equation, shows good agreement with the FCS and molecular dynamics simulations.

This data represents the first direct comparison of viscosities calculated for lipid bilayers from the fluorescence lifetimes of molecular rotors with established methods of investigating the physical properties of bilayers. The data suggests that the lifetimes of the BODIPY rotors provide an accurate portrayal of membrane "viscosity", and that the methanol-glycerol mixtures are indeed a suitable calibration system.

\section{Viscosity studies in giant unilamellar vesicles}

Giant unilamellar vesicles (GUVs) provide a useful model system to mimic the plasma membranes of cells, as they can be easily made with a variety of compositions and are on the same order of size as cells, allowing imaging using optical microscopy. To evaluate the practicality of using the three rotors as probes of phase separation within lipid bilayers, we prepared phase-separating ternary GUVs made up of mixtures of DOPC:EYSM:Chol using electroformation.

Confocal images of ternary GUVs showing $\mathrm{L}_{\mathrm{o}}-\mathrm{L}_{\mathrm{d}}$ phase separation stained with the three rotors are shown in Fig. 6, with all three probes showing a strong preference for the $L_{d}$ phase.

Whilst rotor 1 has previously been shown to partition poorly into the $\mathrm{L}_{\mathrm{o}}$ phase, ${ }^{19}$ it is perhaps surprising that rotor 3 with its cholestanol derivative also shows a poor partitioning into the $\mathrm{L}_{\mathrm{o}}$ phase. This could be explained by the presence of the phenyl rings on each of the rotors which are not typically present in the $\mathrm{L}_{\mathrm{o}}$ targeting BODIPY dyes. The phenyl ring is bulky and

Table 1 Diffusion coefficients calculated for the three rotors in DOPC bilayers using molecular dynamics simulations, FCS and the Saffman-Delbrück equation to calculate diffusion coefficients from the viscosity values calculated from the fluorescence lifetimes of the BODIPY rotors. Further information

\begin{tabular}{|c|c|c|c|c|c|c|c|c|c|}
\hline \multirow[b]{3}{*}{ Temperature/K } & \multicolumn{9}{|c|}{ Diffusion coefficient $/ \mu \mathrm{m}^{2} \mathrm{~s}^{-1}$} \\
\hline & \multicolumn{3}{|l|}{ Rotor 1} & \multicolumn{3}{|l|}{ Rotor 2} & \multicolumn{3}{|l|}{ Rotor 3} \\
\hline & Simulated & FCS & Lifetime & Simulated & FCS & Lifetime & Simulated & FCS & Lifetime \\
\hline 293 & 10.22 & 10.52 & 3.78 & 7.52 & 8.75 & 2.91 & 7.06 & 7.25 & 3.23 \\
\hline 313 & 16.45 & 18.06 & 11.11 & 14.39 & 12.32 & 8.73 & 12.20 & 14.18 & 8.21 \\
\hline 333 & 22.67 & 22.65 & 32.94 & 21.61 & 19.91 & 27.77 & 18.74 & 21.18 & 20.52 \\
\hline
\end{tabular}
on the simulated diffusion coefficients can be found in Table S1 and Fig. S1, ESI 

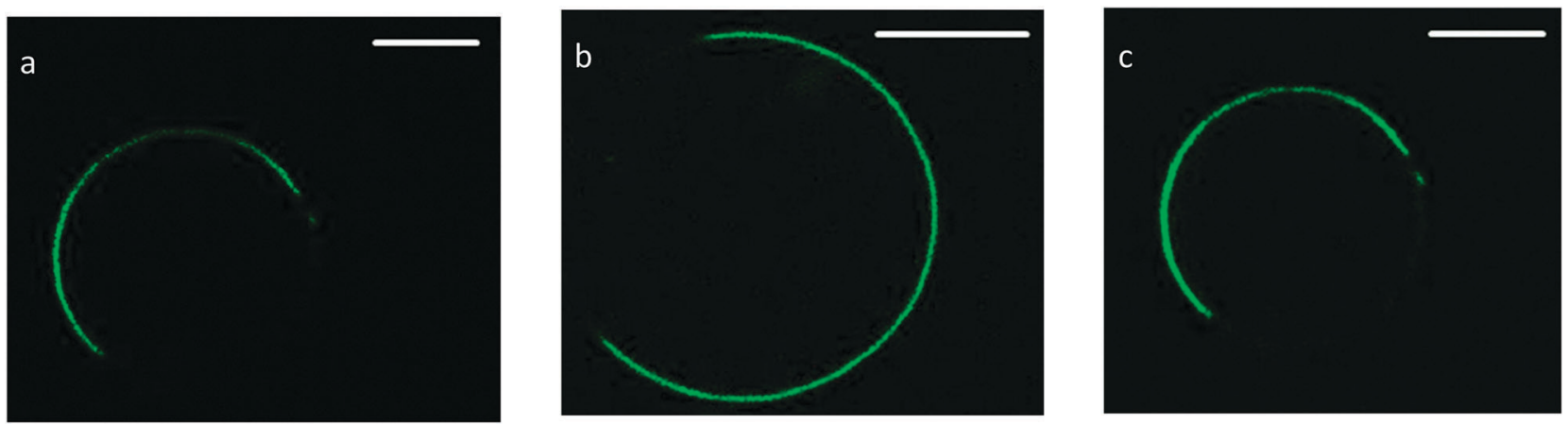

Fig. 6 Confocal images of $L_{o}-L_{d}$ phase separated DOPC/EYSM/Chol GUVs with (a) rotor 1, (b) rotor 2 and (c) rotor $\mathbf{3}$. Scale bar $=10 \mu \mathrm{m}$.

not co-planar with the BODIPY core, and as such is a likely reason for poor $\mathrm{L}_{\mathrm{o}}$ partitioning. Cholesterol-conjugated BODIPY dyes without a phenyl group have been $\operatorname{shown}^{33,53}$ to display much greater partitioning into the $\mathrm{L}_{\mathrm{o}}$ phase, although this has not yet been shown for BODIPY molecular rotors.

Despite the large difference in BODIPY concentration between the two phases, it was possible to obtain fluorescence lifetime images of BODIPY in both $\mathrm{L}_{\mathrm{o}}$ and $\mathrm{L}_{\mathrm{d}}$ phases. Since fluorescence lifetime of BODIPY rotors (and hence, FLIM) is concentrationindependent, ${ }^{15}$ we were able to extract reliable viscosity values for each of the phases imaged. The preferential partitioning of the rotors into the $\mathrm{L}_{\mathrm{d}}$ phase may however limit the usefulness of BODIPY based rotors in the study of lipid order within complex and live biological membrane systems, where $\mathrm{L}_{\mathrm{O}}$-like domains are likely to be small and short lived. ${ }^{54}$

The phase diagram for a ternary mixture of DOPC:EYSM: $\mathrm{Chol}^{55}$ (Fig. S11, ESI $\dagger$ ) highlights the $\mathrm{L}_{\mathrm{O}}-\mathrm{L}_{\mathrm{d}}$ phase coexistence region and the tie lines across this region. At the critical point the compositions of the $\mathrm{L}_{\mathrm{o}}$ and $\mathrm{L}_{\mathrm{d}}$ phases are identical, and near this point their compositions should be similar, meaning the $\mathrm{L}_{\mathrm{o}}$ phase is more disordered in character, and will likely have a similar viscosity to the $\mathrm{L}_{\mathrm{d}}$ phase. This should also allow the BODIPY probes to partition to a greater extent into the $\mathrm{L}_{\mathrm{o}}$ phase. Using BODIPY rotor 1 for imaging, GUVs composed of four different ratios of DOPC : EYSM : Chol (A - 56:24:20, B - $40: 25: 35, \mathrm{C}-48: 40: 12$ and $\mathrm{D}-20: 55: 25)$ were produced along two different tie lines in the phase diagram. As they are located on the same tie lines, mixtures $A$ and $B$ should have $L_{o}$ and $L_{d}$ phases of the same compositions, but in different ratios. This should also be true for mixtures C and D. FLIM was used to determine the lifetime, and therefore the viscosity, of the four different GUV compositions at room temperature (Fig. 7). The mean viscosities determined for the $L_{o}$ and $L_{d}$ phases are shown in Table 2.

The FLIM images in Fig. 7 are plotted in the same colour scale, from 1500 to $3200 \mathrm{ps}$. The lifetimes of the $\mathrm{L}_{\mathrm{o}}$ phases in A are very similar (within $10 \%$ ) to those in $\mathrm{B}$, and the $\mathrm{L}_{\mathrm{d}}$ phases of $\mathrm{A}$ and $\mathrm{B}$ are also very similar. A similar observation is made for the lifetimes of the $\mathrm{L}_{\mathrm{o}}$ and $\mathrm{L}_{\mathrm{d}}$ phases in GUVs $\mathrm{C}$ and $\mathrm{D}$, which lie on a different tie-line. Histograms showing the lifetime distributions of the four vesicles shown in Fig. 7 can be found in Fig. S12 (ESI $\dagger$ ). On the phase diagram, the $\mathrm{L}_{\mathrm{d}}$ compositions for $\mathrm{A}, \mathrm{B}, \mathrm{C}$ and $\mathrm{D}$ are close together, hence their similar viscosities. However, the $\mathrm{L}_{\mathrm{o}}$ composition of $\mathrm{A}$ and $\mathrm{B}$ is significantly different to that for $\mathrm{C}$ and $\mathrm{D}$, which is highlighted by their different viscosities.
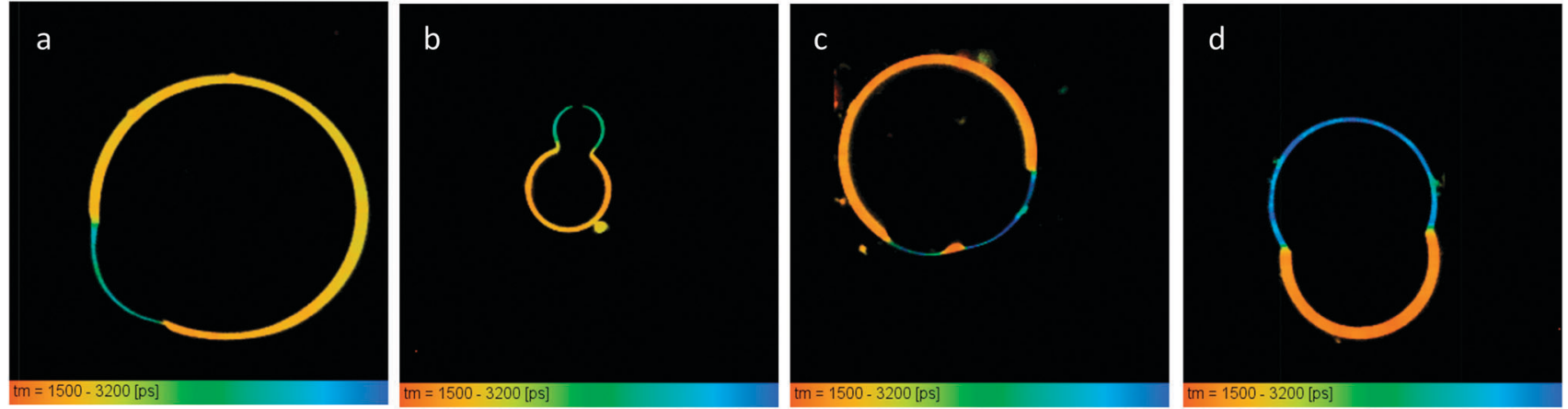

Fig. 7 Representative fluorescence lifetime images of GUV from lipid mixtures A, B, C and D, imaged with rotor 1 . The lipid compositions of GUVs A and $B$ lie on the same tie line within the DOPC: EYSM: Chol phase diagram (see Fig. S11, ESI $\dagger$ ), hence the compositions and therefore viscosities of the $L_{o}$ (blue/green) and $L_{d}$ (orange) phases in GUVs $A$ and $B$ are very similar, which is reflected in the fluorescence lifetimes. The same is true for GUVs $C$ and $D$, however they lie on a different tie line to GUVs $A$ and $B$ and this results in a significant difference in the $L_{\circ}$ phase lifetime between these two pairs of vesicles. The fluorescence lifetime range 1500-3200 ps represents a viscosity range of $94.6-454.3 \mathrm{cP}$. Note that the bulging of the different phases is a result of the difference in membrane thickness and molecular motion between the $L_{o}$ and $L_{d}$ phases, which leads to a line tension between these membrane regions and an energetic drive to minimise the length of the line interface. ${ }^{52}$ Histograms corresponding to these lifetime images can be found in Fig. S12 (ESI $\dagger$ ). 
Table 2 Room temperature viscosities of $L_{\circ}$ and $L_{d}$ phases obtained for GUV compositions $\mathrm{A}, \mathrm{B}, \mathrm{C}$ and $\mathrm{D}$ by applying the calibration to the FLIM data for $\mathbf{1}^{a}$

\begin{tabular}{lll}
\hline Composition & Mean $\mathrm{L}_{\mathrm{d}}$ viscosity/cP & Mean $\mathrm{L}_{\mathrm{o}}$ viscosity/cP \\
\hline A & 128.9 & 251.1 \\
$\mathrm{~B}$ & 134.7 & 227.3 \\
$\mathrm{C}$ & 124.4 & 333.2 \\
$\mathrm{D}$ & 125.4 & 336.7
\end{tabular}

${ }^{a}$ Calculated as an average of several GUV lifetime images - see Fig. S13 (ESI) for the lifetimes and error bars used to calculate these values.

These calculated viscosity values are in good agreement with the viscosity values calculated in Fig. $4 \mathrm{c}$ and $\mathrm{d}$ for DOPC and SPM bilayers containing cholesterol.

\section{Conclusions}

In conclusion, we report the synthesis of a novel cholestanol derivative of a BODIPY molecular rotor. We compared the use of three different BODIPY-based molecular rotors as probes of viscosity within artificial bilayers. We investigated the effects of cholesterol on the viscosity of saturated and unsaturated lipids within model bilayers and the viscous properties of a saturated lipid bilayer above and below the gel transition temperature. Whilst the rotor 3 is shown not to specifically target the $\mathrm{L}_{\mathrm{o}}$ phase in GUVs, it may yet offer significant advantages for in vitro and in vivo targeting.

Through the use of molecular dynamics simulations, we investigated the orientation of the BODIPY rotors within lipid bilayers, which revealed that the rotors take on one preferred orientation in fluid phase bilayers and two orientations in gel phase bilayers. The latter is likely to give rise to the observed biexponential fluorescence decays of the rotors in the gel phase. In addition to this, we used molecular dynamics simulations in conjunction with FCS to determine the diffusion coefficients of the BODIPY rotors within DOPC bilayers at a range of temperatures. By comparing these diffusion coefficients with diffusion coefficients derived, via the Saffman-Delbrück equation, from the viscosity values obtained from the fluorescence lifetimes of the three rotors in DOPC bilayers, we verified the accuracy of using the fluorescence lifetime of molecular rotors to investigate the viscous properties of lipid bilayers. To the best of our knowledge, this is the first study to compare these three methods of determining membrane fluidity.

Finally, we demonstrated the use of BODIPY based molecular rotors as probes of phase separation in bilayer systems, confirming that the $\mathrm{L}_{\mathrm{o}}$ phase has a higher viscosity than the $\mathrm{L}_{\mathrm{d}}$ phase. Whilst in principle this could provide a useful tool in investigating the presence of ordered microdomains in cellular plasma membranes, we note that the three probes analysed partition strongly into the $\mathrm{L}_{\mathrm{d}}$ phase, potentially limiting their usefulness as probes of phase behaviour in cellular plasma membranes. The next synthetic challenge lies with the design and synthesis of a BODIPY rotor probe that partitions significantly into the $\mathrm{L}_{\mathrm{o}}$ phase, as well as into the plasma membrane of a living cell, with low rates of endocytosis.

\section{Acknowledgements}

MKK and JAB are thankful to the UK's Engineering and Physical Science Research Council (EPSRC) for their Career Acceleration Fellowships. NJB is thankful for the EPSRC grant EP/J017566/1. NDG is thankful for the Proxomics grant EP/I017887/1. IRG would like to acknowledge funding from the EU in the form of the project "HeCaToS - Hepatic and Cardiac Toxicology Systems modelling" FP7-HEALTH-2013-INNOVATION-1 (Project number 60216). RK is thankful to the BHF programme grant no. RG/11/13/29055. JMC wishes to acknowledge the support of the ERC Advanced Fellowship, Bio-Phononics (340117). We are thankful to Craig Young for use of the rheometer in determining the viscosities of castor oil and the viscous diols at a range of temperatures.

\section{Notes and references}

1 M. K. Kuimova, Phys. Chem. Chem. Phys., 2012, 14, 12671-12686.

2 A. G. Lee, Biochim. Biophys. Acta, 2004, 1666, 62-87.

3 G. E. Atilla-Gokcumen, E. Muro, J. Relat-Goberna, S. Sasse, A. Bedigian, M. L. Coughlin, S. Garcia-Manyes and U. S. Eggert, Cell, 2014, 156, 428-439.

4 V. V Shynkar, A. S. Klymchenko, C. Kunzelmann, G. Duportail, C. D. Muller, A. P. Demchenko, J.-M. Freyssinet and Y. Mely, J. Am. Chem. Soc., 2007, 129, 2187-2193.

5 P. K. Ghosh, A. Vasanji, G. Murugesan, S. J. Eppell, L. M. Graham and P. L. Fox, Nat. Cell Biol., 2002, 4, 894-900.

6 J. Diao, T. Y. Yoon, Z. Su, Y. K. Shin and T. Ha, Langmuir, 2009, 25, 7177-7180.

7 J. Diao, D. J. Cipriano, M. Zhao, Y. Zhang, S. Shah, M. S. Padolina, R. A. Pfuetzner and A. T. Brunger, J. Am. Chem. Soc., 2013, 135, 15274-15277.

8 S.-T. Yang, V. Kiessling, J. A. Simmons, J. M. White and L. K. Tamm, Nat. Chem. Biol., 2015, 11, 424-431.

9 A. K. Kenworthy, B. J. Nichols, C. L. Remmert, G. M. Hendrix, M. Kumar, J. Zimmerberg and J. LippincottSchwartz, J. Cell Biol., 2004, 165, 735-746.

10 T. J. Feder, I. Brust-Mascher, J. P. Slattery, B. Baird and W. W. Webb, Biophys. J., 1996, 70, 2767-2773.

11 V. Mueller, C. Ringemann, A. Honigmann, G. Schwarzmann, R. Medda, M. Leutenegger, S. Polyakova, V. N. Belov, S. W. Hell and C. Eggeling, Biophys. J., 2011, 101, 1651-1660.

12 M. A. Haidekker and E. A. Theodorakis, Org. Biomol. Chem., 2007, 5, 1669-1678.

13 T. Förster and G. Hoffmann, Z. Phys. Chem., 1971, 75, 63-76. 14 M. K. Kuimova, S. W. Botchway, A. W. Parker, M. Balaz, H. A. Collins, H. L. Anderson, K. Suhling and P. R. Ogilby, Nat. Chem., 2009, 1, 69-73.

15 M. A. Haidekker, T. P. Brady, D. Lichlyter and E. A. Theodorakis, J. Am. Chem. Soc., 2006, 128, 398-399.

16 F. Liu, T. Wu, J. Cao, S. Cui, Z. Yang, X. Qiang, S. Sun, F. Song, J. Fan, J. Wang and X. Peng, Chem. - Eur. J., 2013, 19, 1548-1553.

17 Z. Yang, J. Cao, Y. He, J. H. Yang, T. Kim, X. Peng and J. S. Kim, Chem. Soc. Rev., 2014, 43, 4563-4601. 
18 J. A. Levitt, M. K. Kuimova, G. Yahioglu, P. H. Chung, K. Suhling and D. Philips, J. Phys. Chem. C, 2009, 113, 11634-11642.

19 Y. Wu, M. Štefl, A. Olzyńska, M. Hof, G. Yahioglu, P. Yip, D. R. Casey, O. Ces, J. Humpolíčková and M. K. Kuimova, Phys. Chem. Chem. Phys., 2013, 15, 14986-14993.

20 I. López-Duarte, T. T. Vu, M. A. Izquierdo, J. A. Bull and M. K. Kuimova, Chem. Commun., 2014, 50, 5282-5284.

21 M. K. Kuimova, G. Yahioglu, J. A. Levitt and K. Suhling, J. Am. Chem. Soc., 2008, 130, 6672-6673.

22 K. Suhling, P. M. W. French and D. Phillips, Photochem. Photobiol. Sci., 2005, 4, 13-22.

23 M. A. H. Alamiry, A. C. Benniston, G. Copley, K. J. Elliott, A. Harriman, B. Stewart and Y.-G. Zhi, Chem. Mater., 2008, 20, 4024-4032.

24 E. Bahaidarah, A. Harriman, P. Stachelek, S. Rihn, E. Heyer and R. Ziessel, Photochem. Photobiol. Sci., 2014, 13, 1397-1401.

25 N. Boens, V. Leen and W. Dehaen, Chem. Soc. Rev., 2012, 41, 1130-1172.

26 H. L. Kee, C. Kirmaier, L. Yu, P. Thamyongkit, W. J. Youngblood, M. E. Calder, L. Ramos, B. C. Noll, D. F. Bocian, W. R. Scheidt, R. R. Birge, J. S. Lindsey and D. Holten, J. Phys. Chem. B, 2005, 109, 20433-20443.

27 N. A Hosny, G. Mohamedi, P. Rademeyer, J. Owen, Y. Wu, M.-X. Tang, R. J. Eckersley, E. Stride and M. K. Kuimova, Proc. Natl. Acad. Sci. U. S. A., 2013, 110, 9225-9230.

28 P. Loison, N. A. Hosny, P. Gervais, D. Champion, M. K. Kuimova and J. M. Perrier-Cornet, Biochim. Biophys. Acta, 2013, 1828, 2436-2443.

29 H. Zhu, J. Fan, M. Li, J. Cao, J. Wang and X. Peng, Chem. Eur. J., 2014, 20, 4691-4696.

30 Z. Li and R. Bittman, J. Org. Chem., 2007, 72, 8376-8382.

31 Z. Li, E. Mintzer and R. Bittman, J. Org. Chem., 2006, 71, 1718-1721.

32 J. O. Flores-Rizo, I. Esnal, C. A. Osorio-Martínez, C. F. A. Gómez-Durán, J. Bañuelos, I. L. Arbeloa, K. H. Pannell, A. J. Metta-Magaña and E. Peña-Cabrera, J. Org. Chem., 2013, 78, 5867-5877.

33 L. M. Solanko, A. Honigmann, H. S. Midtiby, F. W. Lund, J. R. Brewer, V. Dekaris, R. Bittman, C. Eggeling and D. Wüstner, Biophys. J., 2013, 105, 2082-2092.

34 T. A. Daly, M. Wang and S. L. Regen, Langmuir, 2011, 27, 2159-2161.

35 M. E. Beattie, S. L. Veatch, B. L. Stottrup and S. L. Keller, Biophys. J., 2005, 89, 1760-1768.

36 A. Loudet and K. Burgess, Chem. Rev., 2007, 107, 4891-4932.

37 L. A. Bagatolli, T. Parasassi and E. Gratton, Chem. Phys. Lipids, 2000, 105, 135-147.
38 D. A. Case, T. A. Darden, T. E. Cheatham III, C. L. Simmerling, J. Wang, R. E. Duke, R. Luo, R. C. Walker, W. Zhang, K. M. Merz, B. Robert, S. Hayik, A. Roitberg, G. Seabra, J. Swails, A. W. Goetz, I. Kolossvary, K. F. Wong, F. Paesani, J. Vanieck, R. M Wolf, J. Liu, X. Wu, S. R. Brozell, T. Steinbrecher, H. Gohlke, Q. Cai, X. Ye, J. Wang, M.J. Hsieh, G. Cui, D. R. Roe, D. H. Mathews, M. G. Seetin, R. Salomen-Ferrer, C. Sagui, V. Babin, T. Luchko, S. Gusarov, A. Kovalenko and P. A. Kollman, AMBER 12, University of California, San Francisco, 2012.

39 C. J. Dickson, B. D. Madej, Å. A. Skjevik, R. M. Betz, K. Teigen, I. R. Gould and R. C. Walker, J. Chem. Theory Comput., 2014, 10, 865-879.

40 J. Enderlein, I. Gregor, D. Patra and J. Fitter, Curr. Pharm. Biotechnol., 2004, 5, 155-161.

41 M. A. Haidekker, T. P. Brady, D. Lichlyter and E. A. Theodorakis, Bioorg. Chem., 2005, 33, 415-425.

42 D. M. Owen, D. J. Williamson, A. Magenau and K. Gaus, Nat. Commun., 2012, 3, 1256.

43 K. Gaus, E. Chklovskaia, B. F. de St. Groth, W. Jessup and T. Harder, J. Cell Biol., 2005, 171, 121-131.

44 M. Olšinová, P. Jurkiewicz, M. Pozník, R. Sachl, T. Prausová, M. Hof, V. Kozmík, F. Teplý, J. Svoboda and M. Cebecauer, Phys. Chem. Chem. Phys., 2014, 16, 10688-10697.

45 H. Ohvo-Rekilä, B. Ramstedt, P. Leppimäki and J. Peter Slotte, Prog. Lipid Res., 2002, 41, 66-97.

46 D. A. Mannock, T. J. McIntosh, X. Jiang, D. F. Covey and R. N. McElhaney, Biophys. J., 2003, 84, 1038-1046.

47 T. T. Mills, J. Huang, G. W. Feigenson and J. F. Nagle, Gen. Physiol. Biophys., 2009, 28, 126-139.

48 P. A. Hyslop, B. Morel and R. D. Sauerheber, Biochemistry, 1990, 29, 1025-1038.

49 A. M. Seddon, D. Casey, R. V. Law, A. Gee, R. H. Templer and O. Ces, Chem. Soc. Rev., 2009, 38, 2509-2519.

50 R. Macháň and M. Hof, Biochim. Biophys. Acta, 2010, 1798, 1377-1391.

51 P. G. Saffman and M. Delbrück, Proc. Natl. Acad. Sci. U. S. A., 1975, 72, 3111-3113.

52 A. R. Honerkamp-Smith, P. Cicuta, M. D. Collins, S. L. Veatch, M. den Nijs, M. Schick and S. L. Keller, Biophys. J., 2008, 95, 236-246.

53 F. S. Ariola, Z. Li, C. Cornejo, R. Bittman and A. A. Heikal, Biophys. J., 2009, 96, 2696-2708.

54 C. Eggeling, C. Ringemann, R. Medda, G. Schwarzmann, K. Sandhoff, S. Polyakova, V. N. Belov, B. Hein, C. von Middendorff, A. Schönle and S. W. Hell, Nature, 2009, 457, 1159-1162.

55 N. Bezlyepkina, R. S. Gracià, P. Shchelokovskyy, R. Lipowsky and R. Dimova, Biophys. J., 2013, 104, 1456-1464. 